\title{
Creating vortex rings and three-dimensional skyrmions in Bose-Einstein condensates
}

\author{
J. Ruostekoski ${ }^{1}$ and J. R. Anglin ${ }^{2}$ \\ ${ }^{1}$ Department of Physical Sciences, University of Hertfordshire, Hatfield, Herts, AL10 9AB, UK \\ ${ }^{2}$ Institute for Theoretical Atomic and Molecular Physics, \\ Harvard-Smithsonian Center for Astrophysics, Cambridge MA 02135
}

(October 29, 2018)

We propose a method of generating a vortex ring in a BoseEinstein condensate by means of electromagnetically-induced atomic transitions. Some remnant population of atoms in a second internal state remains within the toroidal trap formed by the mean field repulsion of the vortex ring. This population can be removed, or it can be made to flow around the torus (i.e. within the vortex ring). If this flow has unit topological winding number, the entire structure formed by the two condensates is an example of a three-dimensional skyrmion texture.

03.75.Fi,05.30.Jp

With the recent controlled creation of vortices [1.2] in trapped atomic Bose-Einstein condensates (BECs), and evidence for vortex nucleation above a critical flow velocity [3], the dynamics of topological structures in weakly interacting superfluids has become an active subject of experimental research. In this paper we propose methods of preparing a vortex ring within a BEC, allowing the controlled study of a vortex structure away from surface effects 4. The vortex ring is created by means of driving electromagnetic (em) fields inducing transitions between internal atomic levels. The em field couples to the relative phases between the levels, in such a way as to transfer angular momentum to the atoms. With an appropriately chosen superposition of em fields the local axes of this effective rotation form a closed circular loop, producing a vortex ring in the BEC. We also consider the stability of the vortex ring, and identify a way to use a second atomic component of the BEC to increase the stability of the ring against collapsing under its own string tension. Conversely, the vortex ring acts as a trap for the second component, providing purely atomoptical confinement of cold atoms. Finally, the combined two-component structure may be identified as a threedimensional (3D) skyrmion [5], shown in Fig. \&. This nonsingular texture is of interest in its own right, as a topological structure beyond the simple vortex.

Topological defects such as vortices and monopoles possess cores at which the order parameter is singular, and indeed such defects are characterized by winding numbers defined on paths or surfaces that enclose the singular cores. The simplest topological defect relevant to superfluid physics is that of a current flowing around a closed 1D path parametrized by the angular coordinate $\phi$. The simple mapping $\alpha(\phi)=\phi$, from physical space into order parameter space, where $\phi$ is defined on a 1D circle, has 'winding number' one, which will be unchanged if $\alpha(\phi)$ is continuously deformed. Topologically nontrivial configurations are also possible without singular cores: Textures are defined by the way in which the compact order parameter space is 'stretched over' physical space. An $\mathrm{SU}(2)$ order parameter, for instance, takes values on a sphere. One can 'puncture' this sphere, stretch the resulting hole to infinity, and so 'spread' the order parameter space over a 2D plane. Such a mapping, or any continuous deformation of it, is known as a $2 \mathrm{D}$ (or 'baby') skyrmion. Interpreting the order parameter sphere as the spin orientation of a spinor condensate, one can realize a 2D skyrmion as a coreless point vortex (or in $3 \mathrm{D}$, a coreless vortex line) [6]7], such as has been created experimentally at JILA [1].
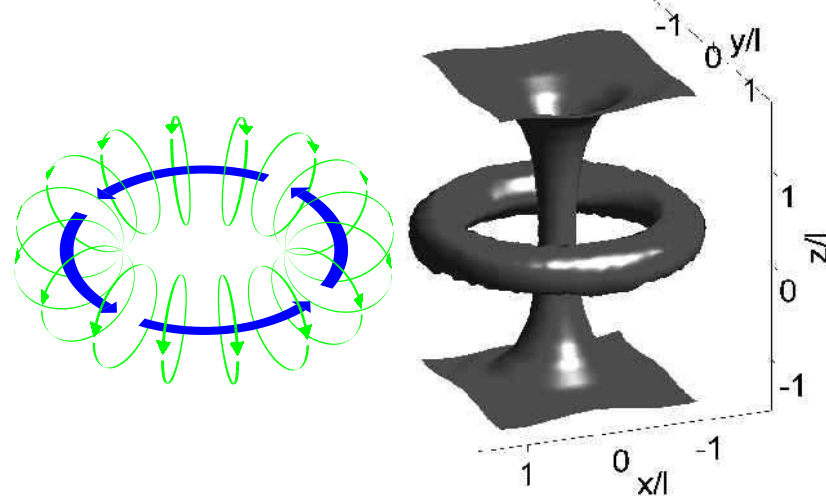

FIG. 1. Schematic illustration of a 3D skyrmion (left), as a vortex ring containing a superflow, and a constant surface plotting of the 3D skyrmion (right) obtained from the numerical simulations. We show the core region of the vortex line and the ring by displaying the atom densities for $\left|\psi_{1}\right|^{2}=\left|\psi_{2}\right|^{2}=1.2 \times 10^{-4} / l^{3}$.

The full, 3D skyrmion results from taking an $S^{3}$ order parameter space and stretching it over $R^{3}$ in a similar way [8]. An $S^{3}$ order parameter space is afforded most simply by a two-component BEC, whose interactions effectively fix the total density $\left|\psi_{1}\right|^{2}+\left|\psi_{2}\right|^{2}=\rho$ of the two complex macroscopic wave functions $\psi_{1}, \psi_{2}$. The low energy degrees of freedom therefore reside on a 3 -sphere of angles $0<\alpha, \beta \leq \pi, 0<\gamma \leq 2 \pi$ (a 3 -sphere is a $3 \mathrm{D}$ subset in $4 \mathrm{D}$, such that $\sum_{n=1}^{4} x_{n}^{2}=R^{2}$ ). For spherical polar coordinates in physical space, a 3D skyrmion is given by any continuous deformation of the mapping $\gamma(r, \theta, \phi)=\phi, \beta(r, \theta, \phi)=\theta, \alpha(r, \theta, \phi)=\lambda(r)$ for monotonic $\lambda$ with $\lambda(0)=0, \lambda(\infty)=\pi$. The topologically 
invariant winding number

$$
W=\frac{1}{2 \pi^{2}} \int d^{3} x \sin ^{2} \alpha \sin \beta \operatorname{det}\left(\frac{\partial \alpha^{i}}{\partial x^{j}}\right),
$$

which obviously vanishes for the ground state of the twospecies BEC, equals one for the skyrmion. (The topological invariance of $W$ is not hard to understand: because the determinant in the integrand is precisely the Jacobian for the change of variables from $x^{i}=(x, y, z)$ to $\alpha^{i}=(\alpha, \beta, \gamma)$, the integral is simply the volume of the unit 3-sphere, regardless of the precise form of $\alpha^{i}\left(x^{j}\right)$.)

We can obtain a physical picture of the $3 \mathrm{D}$ skyrmion by writing out the macroscopic wave functions:

$$
\begin{aligned}
\left(\begin{array}{c}
\psi_{1}(\mathbf{r}) \\
\psi_{2}(\mathbf{r})
\end{array}\right) & \equiv \sqrt{\rho(r)}\left(\begin{array}{c}
-i \sin \alpha \sin \beta \exp (i \gamma) \\
\cos \alpha-i \sin \alpha \cos \beta
\end{array}\right) \\
& =\sqrt{\rho(r)}\left(\begin{array}{c}
-i \sin [\lambda(r)] \sin \theta \exp (i \phi) \\
\cos [\lambda(r)]-i \sin [\lambda(r)] \cos \theta
\end{array}\right) .
\end{aligned}
$$

With this realization of the skyrmion, $\left|\psi_{1}\right|^{2}$ vanishes on the $z$ axis and at infinity, and is concentrated in a toroidal region. On the other hand, $\left|\psi_{2}\right|^{2}$ vanishes on the circle $\theta=\frac{\pi}{2}, r=\lambda^{-1}\left(\frac{\pi}{2}\right)$. Expanding $\psi_{2}$ about any point on this circle, we find $\psi_{2} \propto \delta r+i \delta \theta$, indicating that the nodal circle of $\psi_{2}$ is a vortex line. Hence component $\psi_{2}$ forms a vortex ring, within whose core $\psi_{1}$ resides and flows azimuthally with winding number one. Of course, any continuous deformations of Eq. (2) will still be a skyrmion, and the two-fluid interpretation of the skyrmion will always provide a useful intuitive picture. Moreover, unlike in the case of wave function monopoles [9], changing the atomic basis that defines the two fluids does not lead to a different picture of the skyrmion, but simply produces the same combination of vortex ring and current, with a different spatial orientation.

We propose a method of creating a 3D skyrmion in a trapped BEC using em fields, in a scheme motivated by viewing the skyrmion as this combination of vortex structures. Since several good methods have been proposed for generating simple vortices, and some of these have already been successfully implemented, engineering the state of $\psi_{1}$ presents no new difficulties of principle. Creating the vortex ring state in $\psi_{2}$ is more of a challenge. Although vortex rings may be formed through instabilities, as of a dark soliton in an insufficiently narrow trap [4], a more controlled method of preparing them may also be desirable, in order to study their rich range of motion and excitation.

We consider em-induced coherent scattering of atoms between different internal levels. By superposing different driving em fields we can construct a Rabi amplitude with a spatially-dependent phase profile. In particular, nodes of the em field amplitude may be topological singularities of the em phase. The coupling of the em field to the phase of the coherent matter wave allows the topological singularities of the em fields to be imprinted on the matter field. We demonstrate the preparation process of topological objects by numerically studying this coherent matter-wave dynamics.

The dynamics of the BECs with the Rabi coupling between the levels $|i\rangle$ and $|j\rangle$ follows from the coupled Gross-Pitaevskii equation (GPE)

$$
i \hbar \dot{\psi}_{i}=\left(H_{i}^{0}+\delta_{i}+\sum_{k} \kappa_{i k}\left|\psi_{k}\right|^{2}\right) \psi_{i}+\hbar \Omega_{i j}^{*} \psi_{j}
$$

Here the kinetic energy and the trapping potential are introduced in $H_{i}^{0}$ :

$$
H_{i}^{0} \equiv-\frac{\hbar^{2}}{2 m} \nabla^{2}+\frac{1}{2} m \omega_{i}^{2}\left(x^{2}+\alpha_{i}^{2} y^{2}+\beta_{i}^{2} z^{2}\right) .
$$

We have also defined the interaction coefficients $\kappa_{i j} \equiv$ $4 \pi \hbar^{2} a_{i j} N / m$. Here $a_{i i}$ denotes the intraspecies scattering length in internal level $|i\rangle$ and $a_{i j}(i \neq j)$ stands for the interspecies scattering length. The Rabi frequency, $\Omega(\mathbf{r})$, describes the strength of the coupling between the two internal levels. The total number of BEC atoms, the atomic mass, and the detuning of the em fields from the resonance are denoted by $N, m$, and $\delta_{j}$, respectively.

Several papers [10 15.7] have proposed methods of injecting vorticity into BECs by means of the interaction between em and matter fields. In our adaptation of these methods to create a 3D skyrmion, the simplest part is the step of producing the 'inside' component $\psi_{1}$ with a quantized circulation about the $z$ axis. This can be achieved by transferring population from an initial internal state $|0\rangle$ using the Rabi frequency $\Omega_{01}(\mathbf{r})$,

$$
\Omega_{01}(\mathbf{r})=\Omega_{0}\left[\sin \left(k_{1} x\right)-i \sin \left(k_{1} y\right)\right] \cos \left(k_{2} z\right),
$$

with a similar set of parameters as in Ref. [15]. In particular, $k_{1}^{-1}$ and $k_{2}^{-1}$ are both larger than the size of the sample. The purpose of the $\cos \left(k_{2} z\right)$ is to confine $\psi_{1}$ along the $z$ direction. Note that in this scheme the Rabi frequency could represent a two-photon transition, with one photon from two orthogonal standing waves in the $x y$ plane and the other from a standing wave along the $z$ direction. Alternatively, the $\cos \left(k_{2} z\right)$ could simply represent collimation of the $x y$ beams, which would drive a single photon transition by themselves. The topological singularity in the em field at $x=y=0$ results in a single quantum of circulation about this axis, in the component $\psi_{1}$, as displayed in Fig. 2. One may also generate additional terms in Eq. (5). For instance, the standing wave along the $z$ axis could be further collimated in the $x y$ plane, limiting the spatial extent of $\psi_{1}$.

We propose two different methods of creating the more complicated part of the skyrmion, the closed vortex ring in $\psi_{2}$. The first is an adaptation of the method of Ref. [1], and makes use of two internal levels and the circulating state $\psi_{1}$ once it has been generated. To add the vortex ring requires a Rabi field coupling from state $|1\rangle$ to state $|2\rangle$, which is a phase-coherent superposition of a standing em field and a Gaussian beam, both along the $z$ axis: 
$\Omega_{12}(\mathbf{r})=\Omega_{0}\left[1-\eta^{2}-\exp \left[-\rho^{2} / \xi^{2}\right]+i A \sin \left(k_{3} z\right)\right]$,

with $A=2 \eta /\left(k_{3} \xi\right)$ and $\rho \equiv\left(x^{2}+y^{2}\right)^{1 / 2}$. We are interested in $k_{3}|z| \ll 1, \rho \ll \xi$, and $\eta \ll 1$ in such a way that $\left|k_{3}(\rho-\eta \xi)\right| \ll 1$. Then we obtain

$$
\begin{aligned}
\Omega_{12}(\mathbf{r}) / \Omega_{0} & \simeq \rho^{2} / \xi^{2}-\eta^{2}+i A k_{3} z \\
& \simeq 2 \eta / \xi(\rho-\eta \xi)+i A k_{3} z \\
& =A k_{3}[(\rho-\eta \xi)+i z] .
\end{aligned}
$$

The phase of $\Omega_{12}(\mathbf{r})$ has a form of the quantized circulation with unit winding number around the closed $\operatorname{ring} \rho=$ $\eta \xi$ and $z=0$. Alternatively, the Gaussian beam resulting in the exponential term in Eq. (6) may also be replaced by two standing em fields: $[\cos (2 x / \xi)+\cos (2 y / \xi)] / 2$.

For this first method, the field of Eq. (5) is tuned away from resonance. A localized shifting field is then applied as in Ref. [1], to 'turn on' the transition in a small region, which is then swept around the trap to create a full vortex ring, and also undo the azimuthal phase winding of the $|1\rangle$ atoms, so that the population shifted into $|2\rangle$ will have no angular momentum about the $z$ axis. While this scheme takes advantage of an already demonstrated technology, it has the disadvantage that it tends to produce a highly excited skyrmion state, which may (as discussed below) then evolve self-destructively.
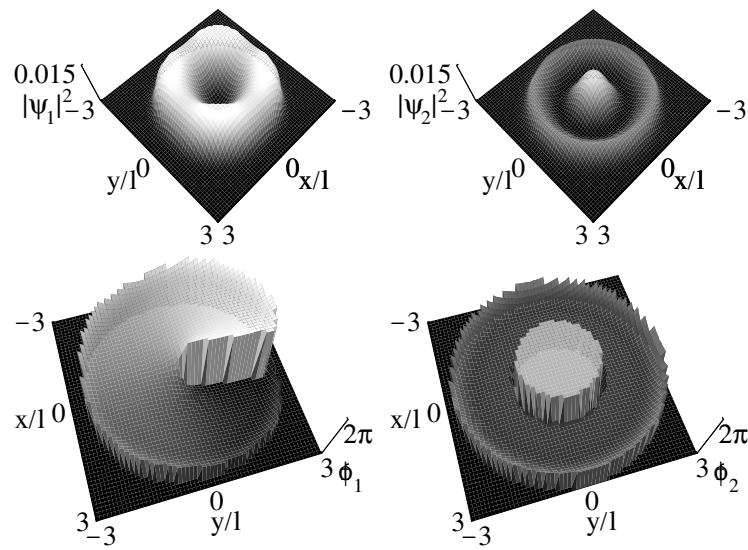

FIG. 2. A 3D skyrmion in the $x y$ plane. We show the density $|\psi(x, y)|^{2}$ and the phase $\phi=\arg [\psi(x, y)]$ profiles of the wave function in the $x y$ plane. Here $\psi(x, y) \equiv \int d z \psi(x, y, z)$. The density profile (upper left) and phase profile (lower left) of atoms in level $|1\rangle$ display a single vortex line with $2 \pi$ phase winding around the core, and vanishing density at the center of the core. The vortex line occupies a toroidal region where the density of the vortex ring of atoms in level $|2\rangle$ (upper right) vanishes. The phase distribution of the vortex ring (lower right) in the $x y$ plane is flat.

We therefore propose, in our second method, to couple atoms with em fields from level $|0\rangle$ to level $|2\rangle$, at the same time as we generate the simple vortex state in level $|1\rangle$. We need a Rabi field coupling from $|0\rangle$ to $|2\rangle$, of the same spatial form as $\Omega_{12}$ of Eq. (6), as well as the $\Omega_{01}$ field of Eq. (5). This scheme requires control of an additional internal state $|0\rangle$, but with a weak coupling it allows adiabatic transfer of the atomic populations [12], producing a ground state skyrmion.

For computational simplicity we demonstrate the three-level preparation process by considering two short nonadiabatic Rabi pulses: The first Rabi pulse of the form (6) generates the vortex ring in level $|2\rangle$ from the initial ground state population in level $|0\rangle$, and then the second pulse with the configuration (5) generates the trapped current in level $|1\rangle$ from the remaining atoms in level $|0\rangle$. Our numerical approach uses three states and can still generate an excited skyrmion, but it is amenable to fully $3 \mathrm{D}$ simulation, and our simulation indicates that the method can work. A careful study of an adiabatic population transfer will require a realistic model for the dissipation of the BECs, as well significant computational resources, and must be left for future investigation.

In our numerical simulations we assume that the traps are isotropic, $\alpha_{i}=\beta_{i}=1$, and that the trapping frequences are equal for all components. We choose the wave number of the em fields $k_{1}=k_{2}=k_{3}=2 \pi /(8 l)$, with $l \equiv[\hbar /(m \omega)]^{1 / 2}, \delta_{i}=0, \eta=0.04, \xi=30 l$, and $\kappa_{i j}=100 \hbar \omega l^{3}$. The number of atoms is determined by the previous relations as $N=25 l /(\pi a)$.

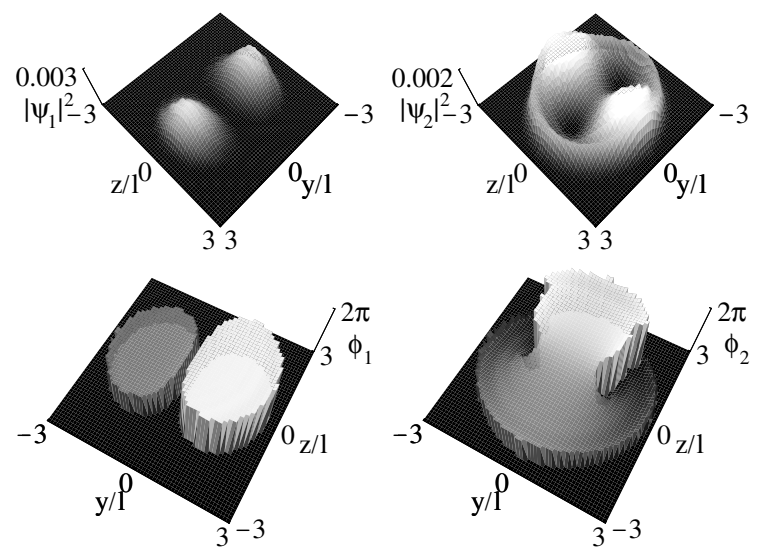

FIG. 3. A 3D skyrmion in the $y z$ plane. We display the density $|\psi(x=0, y, z)|^{2}$ and the phase $\phi=\arg [\psi(x=0, y, z)]$ profiles of the wave function. The density of the single vortex line in level $|1\rangle$ (upper left) occupies the toroidal region surrounded by the vortex ring in level $|2\rangle$ (upper right). The phase profile of the vortex ring (lower right) displays the quantized rotation around the ring.

We integrate the GPE on a spatial grid of $128^{3}$ points, using a split-operator method. The stability of the nonlinear evolution is improved by simultaneously propagating multiple copies of the wave function. Relatively small number of time steps (less than one thousand) provide a sufficient accuracy making the integration executable. Even with up-to-date workstations the optimized simulations can take several hours. Figures 1.3 represent the 3D skyrmion in the case where we have first created the vortex ring in level $|2\rangle$ by applying the Rabi frequency $\Omega_{12}(\mathbf{r})$ [Eq. (6)], with $\Omega_{0}=5000 \omega$ for $0<t \omega \leq 0.04$. This is followed by creating a vortex line in level $|1\rangle$ by the driving $\Omega_{01}$ (r) [Eq. (5)], with $\Omega_{0}=80 \omega$ for $0.04<t \omega \leq 0.05$. 
With the chosen set of parameters a large population remains in $|0\rangle$. This remnant population may be coupled out of the trap immediately after the em pulses as in the recent vortex experiments $[\mathbf{1}$. Due to the shortness of the pulses the interactions of the atoms in level $|0\rangle$ do not have time to affect the structure of the skyrmion. On the other hand, for an efficient adiabatic population transfer the nonlinearity can be a problem. However, the techniques to overcome this were developed in Ref. [12].

The effort of creating such an intricate structure as a vortex ring, let alone a 3D skyrmion, is obviously only justified if its structure can then be observed. As with the filled vortex lines of JILA, the interior BEC in state $|1\rangle$ will enable detection of the density and phase profile of the skyrmion. An additional advantage of the skyrmion, as opposed to an empty vortex ring or to a vortex ring filled with a nonrotating interior BEC, is that it is less unstable against shrinking to zero radius. The kinetic and interaction energy of a vortex line gives it an effective string tension tending to shorten the vortex line, and in the case of a vortex ring, the ring's radius $R$ (not its core thickness!) is energetically unstable to shrinking. In an infinite, homogeneous background, and in the absence of dissipation, the first-order dynamics of a vortex line means that a vortex ring will not actually shrink, but will instead move along its axis, at a velocity determined by its radius. But if its radius approaches its core thickness, a vortex ring can be annihilated (it is not topologically stable), and an excited vortex ring may also possibly twist or pinch, and break up into smaller rings. Moreover, motion at a constant speed cannot continue in a finite, inhomogeneous BEC. Dissipation is also present in experimental conditions, and although straight vortices have been found to have a long lifetime before floating out of the BEC cloud, the energy slope due to string tension may be much steeper than that due to vortex buoyancy (on the healing length scale rather than trap scale). Filling the vortex core lowers the string tension, and hence extends the lifetime of the filled vortex structure in comparison with that of an empty ring.

Although in other field theories skyrmions are often unstable against shrinking to zero size without the topological change of defect nucleation, the separate conservation of both atomic species in our case forbids this decay channel. This does not prevent the vortex ring from collapsing, but it does mean that the component $|1\rangle$ filling the ring cannot be eliminated. Furthermore, since the filling has one unit of angular momentum, there is a centrifugal barrier inhibiting the shrinking. As it tends to pin the vortex ring down, the ring may only collapse via the tunneling of atoms through $\psi_{1}$. Although the vortex rings are dynamically stable [16], numerical evolution in imaginary time shows that filled vortex rings, and even skyrmions, are still energetically unstable, on long enough (imaginary) time scales. Skyrmions are topologically stable as long as the order parameter is well defined everywhere; but they can be destroyed by the nucleation of a defect. Our simulations indicate that, while the en- ergy decreases slowly but monotonically, the vortex ring in $\psi_{2}$ can pull through the state $|1\rangle$ if its inner radius approaches the healing length, so that a healing-length radius circle of zero total density nucleates. However, the decay via the tunneling of atoms through $\psi_{1}$ becomes increasingly slow with the larger occupation in $|1\rangle$ [17. A vortex ring with a nonrotating filling can, if its radius becomes too small, annihilate itself without significantly lowering the total density at any point.

Without dissipation, numerical evolution in real time shows that skyrmions as well as empty vortex rings do move through the background cloud, but instead of simply exiting the cloud as they approach its surface, they generate a background flow in the rest of the cloud which is able to draw them back towards the center. Much more extensive numerical studies will be needed to determine the stability of the resulting oscillation. Further analytical and numerical studies of skyrmion and vortex ring dynamics are clearly warranted, but in this paper we have shown that engineering and study of these advanced BEC structures should be feasible with current techniques.

This research was supported by the NSF and by EPSRC. We thank C.S. Adams, J. Dziarmaga, H.T.C. Stoof, and W.H. Zurek for valuable discussions, and gratefully acknowledge the hospitality of ITAMP at the HarvardSmithsonian Center for Astrophysics.

[1] M.R. Matthews et al., Phys. Rev. Lett. 83, 2498 (1999).

[2] K. Madison et al., Phys. Rev. Lett. 84, 806 (2000).

[3] C. Raman et al., Phys. Rev. Lett. 83, 2502 (1999).

[4] Since our work was completed vortex rings were experimentally created through the instabilities of dark solitons: B.P. Anderson et al., cond-mat/0012444. However, our proposed technique provides a more controlled method of preparing vortex rings.

[5] T.H.R. Skyrme, Proc. Roy. Soc. A 260, 127 (1961); Nucl. Phys. 31, 556 (1962); J. Donoghue et al., Dynamics of the Standard Model, (Cambridge, 1992).

[6] T.-L. Ho, Phys. Rev. Lett. 81, 742 (1998).

[7] K.-P. Marzlin et al., Phys. Rev. A 62, 013602 (2000).

[8] H.T.C. Stoof, cond-mat/0002375.

[9] Th. Busch and J.R. Anglin, Phys. Rev. A60, R2669 (1999).

[10] E.L. Bolda and D.F. Walls, Phys. Lett. A 246, 32 (1998).

[11] K.-P. Marzlin et al., Phys. Rev. Lett. 79, 4728 (1997).

[12] R. Dum et al., Phys. Rev. Lett. 80, 2972 (1998).

[13] J.E. Williams and M.J. Holland, Nature 401, 568 (1999).

[14] L. Dobrek et al., Phys. Rev. A 60, R3381 (1999).

[15] J. Ruostekoski, Phys. Rev. A 61, 041603 (2000).

[16] B. Jackson et al., Phys. Rev. A 61, 013604 (2000).

[17] A very large BEC in level $|1\rangle$ filling the vortex ring may also annihilate the vortex ring by pushing it through the outer boundary of the atomic cloud as a result of the mean-field repulsion. 